\title{
The Combination of Color and Depth Information for Importance Map
}

\author{
Yuhui He \\ Shaanxi Polytechnic Institute, Xianyang, China \\ heyuhui1023@sina.com
}

\begin{abstract}
In order to extract important map, a lot of image resizing algorithms only apply color map (such as gradient or salience information, etc.) to settle this question. On the analysis of depth map, this article thinks about depth information and combines with color map in order to put forward a combined important map. This new important map can identify the main body of source image accurately. Guided by this important map, image resizing results can not only protect the important area, but also obtain better processing effect.
\end{abstract}

Keywords-Depth information; Important map; Combination algorithm; Image resizing

\section{INTRODUCTION}

The image resizing algorithm can be segmented into two phases: the perception phase is the extraction for the importance map on the source image. The accuracy of important map affects the resizing effect directly. For importance map, most resizing algorithm only apply color information (such as gradient or salience information, etc.) to cope with the question. The importance map which is based on color map reflects the focus area of human eye, it is not quite accurately and its accuracy still needs to be improved further. In view of this situation, this paper thinks about depth information which is based on physiology [1] and psychology theory [2-4]. Comparing with other automatic algorithm to calculate the importance map, quantitative results and qualitative analysis show that new algorithm may extract the importance map much more accurate.

\section{COMBINATION EXTRACTION ALGORITHM}

The joint of depth and color information map, the process of the combination extraction algorithm is shown on figure 1. The source information derives from the RGB-D camera. Combination extraction algorithm was divided into four parts: edge test, salience map detection, depth information generation and importance map's extraction. The edge test and salience detection belong to color information processing's category, depth information generation reside in depth information processing's scope, the extraction of importance map not only thinks about the color information in the source image (gradient or salience map), but also combines with the depth map.

\section{A. The Edge Test and Salience Detection}

With regard to edge and saliency test, we use sobel operator [5] to calculate the gradient information and adopt Itti model [6] to detect important map. New algorithm converts color image to grayscale image firstly, and then deals with grayscale image for edge test and saliency detection. Edge test is finished by applying the Sobel model which makes the calculated features keep the continuity on the source image's edge. Sobel model is a discrete difference operator and was applied to label brightness function's approximation on image. In the image's any pixels, we will calculate a corresponding gradient vector by using this operator. This operator contains two sets matrices respectively, they are the horizontal and vertical operator, which was used to deal with the plane convolution on image, we can get the brightness value of the difference approximation.

If the original image is supposed by $I, M_{x}$ and $M_{y}$ are the horizontal edge detection map and vertical map respectively, it will be

$$
M_{x}=\left[\begin{array}{lll}
-1 & 0 & +1 \\
-2 & 0 & +2 \\
-1 & 0 & +1
\end{array}\right] * I, \quad M_{y}=\left[\begin{array}{ccc}
+1 & +2 & +1 \\
0 & 0 & 0 \\
-1 & -2 & -1
\end{array}\right] * I
$$

Among the equation (1), * means the convolution; Each pixel's transverse and longitudinal gradient approximation in the image was calculated by equation (1), then the gradient value $M_{\text {gradient }}$ is get by

$$
M_{\text {gradient }}=\sqrt{M_{x}^{2}+M_{y}^{2}}
$$

And the gradient direction degree $\theta$ is calculated by

$$
\theta=\arctan \left(\frac{M_{y}}{M_{x}}\right)
$$

Supposing $\theta=0$, it means that there has image's vertical edge. Figure 2 (b) is get by applying the Sobel model on the original image (figure 2) (a) to get the gradient information, which reflects the edge character of original image.

For salience test, we use Itti model [6] here. Itti algorithm is based on human vision characteristics, it combines with the theory of psychology and biology, relies on the reaction of human vision to color, brightness, or direction that belong to the underlying characteristics, imitates the human vision reaction to significance information from the bottom to up. Figure 2 (c) is a salient map which is calculated from the Itti algorithm on the original image. Saliency information 
reflects the basic human eyes' focus area, but the gray degree value of face, collar is less than the value of the behind wall. So the information reflected by the salient map is not accurate adequately, it should be optimized further.

\section{B. The Extraction Method for Important map}

Important map is used for guiding the image resizing. For extracting the important map of source information, new algorithm combines the gradient information, salience map and depth information to calculate importance map $M_{\text {depthbased }}$

$$
M_{\text {depthbased }}=\left(M_{\text {gradient }}+M_{\text {saliency }}\right) D_{\text {meanshift }}
$$

$M_{\text {gradient }}$ and $M_{\text {saliency }}$ is the gradient map and salient map, $D_{\text {meanshift }}$ is the depth information. The main purpose of this combination method is to inhibit pixels far away from the camera could get high importance value.

After calculating the important map $M_{\text {depthbased }}$, we could apply this method:

$$
s=c r^{\gamma}
$$

Among equation (5), $r$ is the important map, $s$ is the important map after adjusting the original important map. $C$ and $\gamma$ are the positive parameter respectively. When $\gamma$ is increased, the main body area in image is decreased. When $\gamma$ is decreased, the main body area value is increased. Because the goal of new algorithm is to protect main subject area, we may set $\gamma<1$. Figure 2 shows the example which combines the gradient, saliency information and depth information (figure 2 (d)) to extract the original image's important map (figure 2 (e)) ( $c=1, \gamma=0.4$ is set in this example).

\section{CONCLUSION}

New algorithm in this paper proposes a combination extraction method of color and depth information for importance map, an importance map was get by the combination of color map and depth information. The map that was calculated by new algorithm is more accurate. Resizing result can reduce the deformation of the subject in original image.

\section{REFERENCES}

[1] G.R. Fink, R.J. Dolan, and P.W. Halligan, "Space-based and objectbased visual attention: shared and specific neural domains,” BRAIN, vol. 120(11), pp. 2013-2028, 1997.

[2] C.W. Eriksen, J.E. Hoffman, "The extent of processing of noise elements during selective encoding from visual displays,” Perception and Psychophysics, vol. 14(1), pp. 155-160, 1973.

[3] B.A. Eriksen, C.W. Eriksen, "Effects of noise letters upon the identification of a target letter in a nonsearch task," Perception and Psychophysics, vol. 16(1), pp. 143-149, 1974.

[4] E. Fox, "Perceptual grouping and visual selective attention," Perception and Psychophysics, vol. 60(6), pp. 1004-1021, 1998.

[5] C.G. Rafael, E.W. Richard, "Digital image processing," Second Edition. BEIJING: Publishing House of Electronics Industry, pp. 425463, 2010.

[6] L. Itti, C. Koch, E. Ernst, "A model of saliency-based visual attention for rapid scene analysis," IEEE Transactions on Pattern Analysis and Machine Intelligence, vol. 20(11), pp. 1254-1259, 1998.

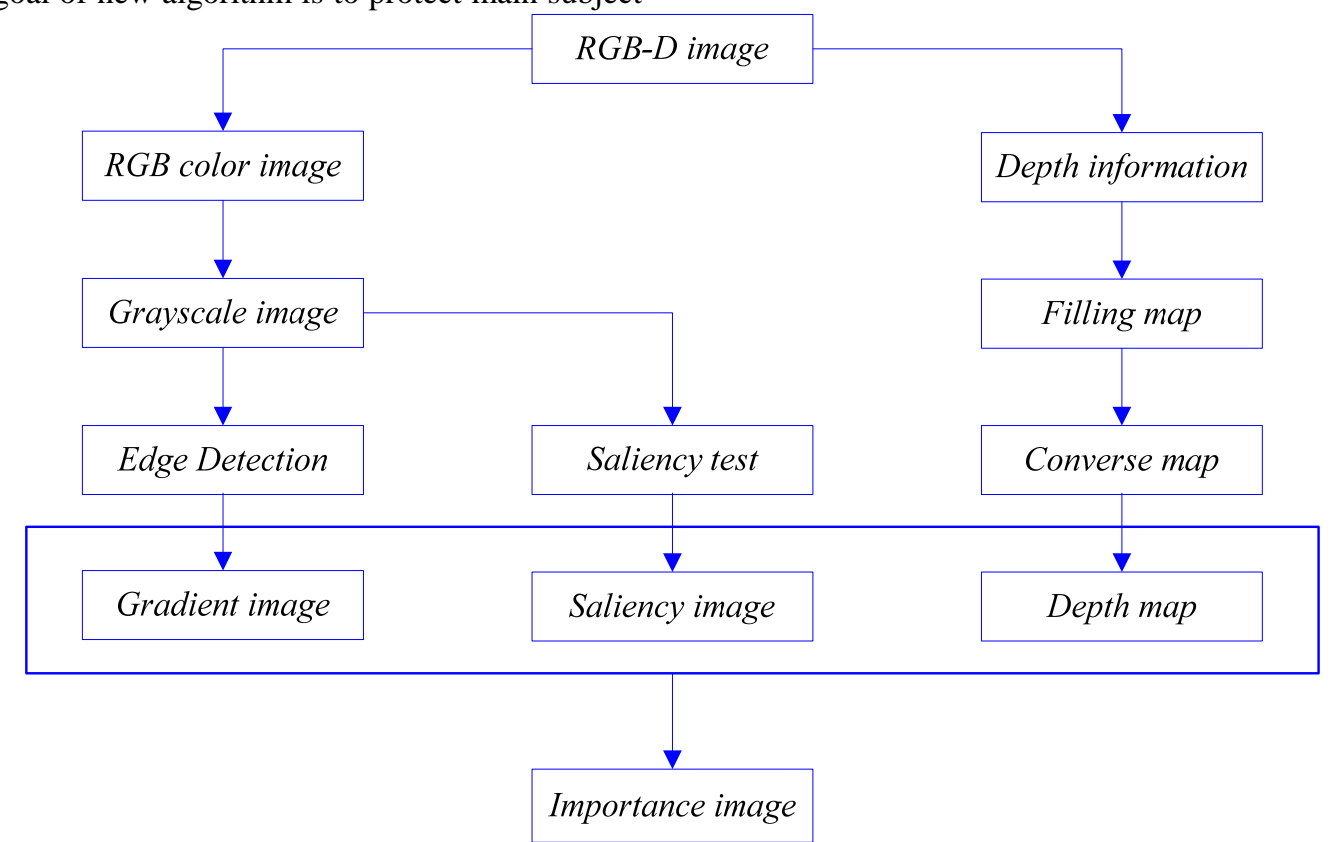

Figure 1. The flow of important map's extraction algorithm 


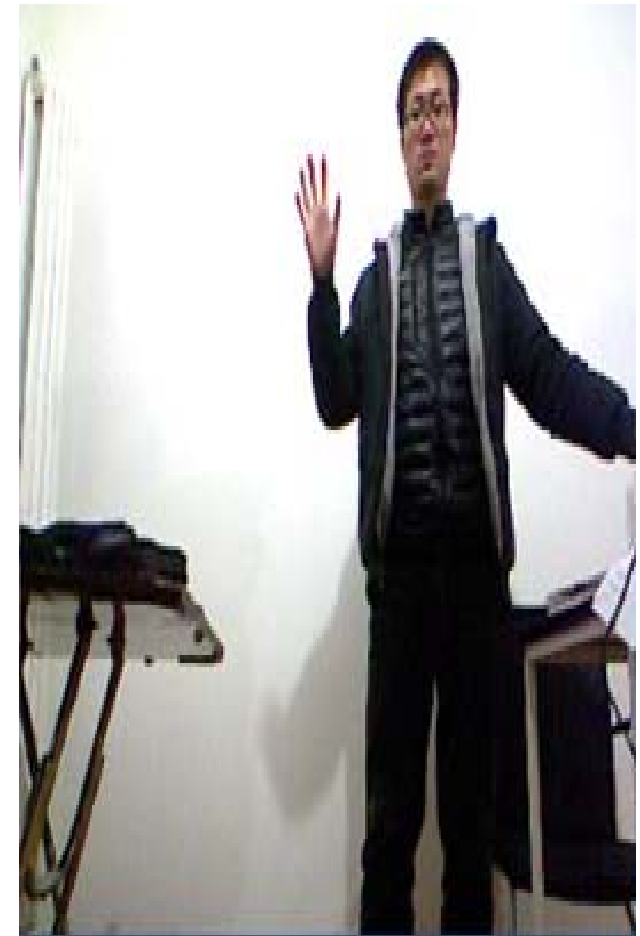

(a) Original image

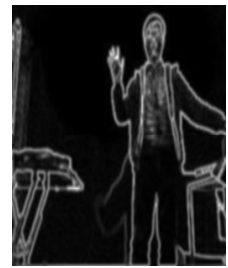

(b) Gradient map

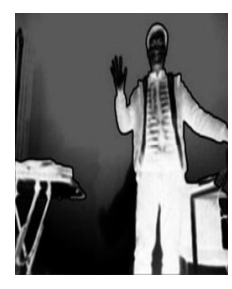

(c) Saliency map

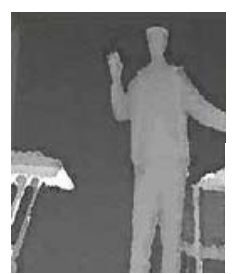

(d) Depth map

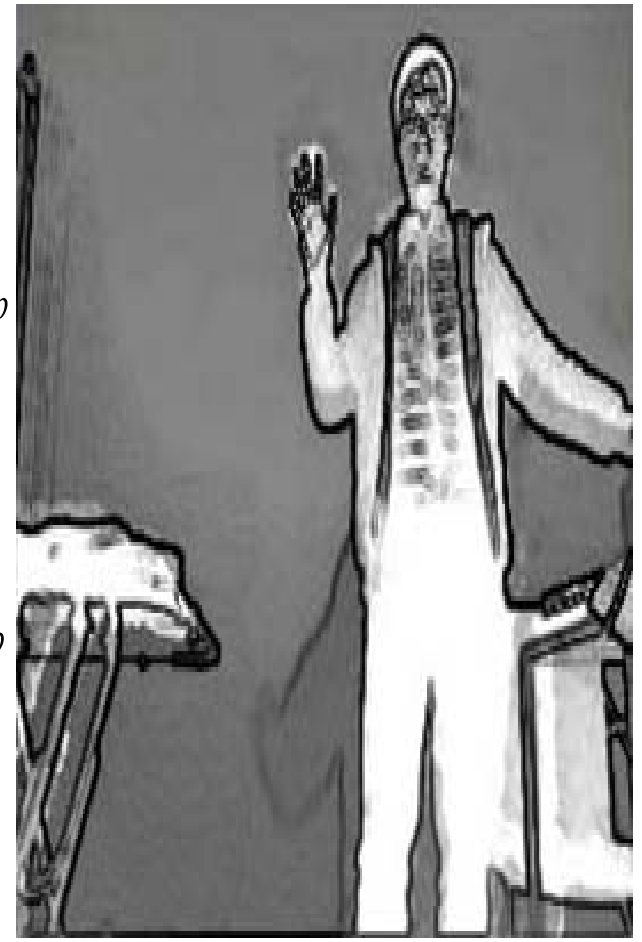

(e) Importance map

Figure 2. The example about important map's calculation ( $c=1, \gamma=0.4$ ) 